NUMBER THEORY WEEK 2017

BANACH CENTER PUBLICATIONS, VOLUME 118

INSTITUTE OF MATHEMATICS

POLISH ACADEMY OF SCIENCES

WARSZAWA 2019

\title{
SOME NEW DENSITY THEOREMS FOR DIRICHLET $L$-FUNCTIONS
}

\author{
JÁNOS PINTZ \\ Rényi Mathematical Institute of the Hungarian Academy of Sciences \\ Reáltanoda u. 13-15, H-1053 Budapest, Hungary \\ E-mail: pintz.janos@renyi.mta.hu
}

Dedicated to Jerzy Kaczorowski on his $60^{\text {th }}$ birthday

\begin{abstract}
We prove some new log-free density theorems for zeros of Dirichlet L-functions (which accordingly are more sharp than earlier ones near to the boundary line of the critical strip). The results can be applied in several problems of prime number theory.
\end{abstract}

1. It is well known that the maximum of the error term of the Prime Number Theorem (or its analogue for primes in an arithmetic progression) depends on the zero-free region of the Riemann's zeta-function (or the Dirichlet's $L$-functions, respectively) or, in other words, on the zeros lying nearest to the boundary line $\operatorname{Re} s=\sigma=1$.

On the other hand, many other arithmetic problems depend not only on the situation of the extreme right-hand zeros but also on the number of such zeros. The first theorem of such type was proved nearly 100 years ago by F. Carlson [1] in 1920. These results were later called density theorems. They proved to be very useful in bounding from above the gaps between consecutive primes (or between consecutive primes in an arithmetic progression) or in Linnik's problem of bounding the first prime in an arithmetic progression.

In some applications the distribution of all zeros $\varrho=\beta+i \gamma$ with $1 / 2 \leq \beta \leq 1$ is important (like in the case of bounding from above gaps between consecutive primes). In other questions only those lying near to the line $\sigma=1$, i.e. with $\beta \geq 1-\varepsilon$ ( $\varepsilon$ small) play a significant role (like in the case of Linnik's constant). In the second type of problems it is

2010 Mathematics Subject Classification: Primary 11N05, 11N36; Secondary 11N35.

Key words and phrases: Hardy-Littlewood prime $k$-tuple conjecture, prime numbers, sieves, gaps between primes, twin primes.

The paper is in final form and no version of it will be published elsewhere. 
also important to prove so-called "log-free" density theorems, where the upper bound for the zeros does not contain any power of the logarithm of the modulus of the relevant arithmetic progression or the logarithm of the height of the relevant zeros.

2. In Section 3 we will prove density theorems in the half-planes $\sigma>3 / 4$ (Theorem 1 ) and $\sigma>1-\varepsilon$ (Theorems 2 and 3), respectively. In the following introduction we will focus on density theorems near the line $\sigma=1$. Let $N(\alpha, T, \chi)$ denote the number of zeros of $L(s, \chi)$ in the rectangle

$$
R(\alpha, T)=\{\sigma+i t ; \alpha \leq \sigma \leq 1,|t| \leq T\}
$$

and let

$$
\begin{aligned}
N(\alpha, T, q) & =\sum_{\chi(\bmod q)} N(\alpha, T, \chi), \\
N^{*}(\alpha, T, Q) & =\sum_{q \leq Q} \sum_{\chi(\bmod q)}^{*} N(\alpha, T, \chi),
\end{aligned}
$$

where the asterisk indicates summation over primitive characters. Fogels [3] and Gallagher [5] proved the first general "log-free" density theorems of the form

$$
N(\alpha, T, q) \ll T^{c(1-\alpha)} \quad \text { for fixed } q \leq T \quad[5
$$

and

$$
N^{*}(\alpha, T, T) \ll T^{c^{\prime}(1-\alpha)} \quad[3
$$

with large values of $c$ and $c^{\prime}$.

Selberg invented a new method — the use of the so-called pseudocharacters (cf. (3.1) — which yielded the estimates [14]:

$$
\begin{aligned}
N(\alpha, T, q) & \ll_{\varepsilon}\left(q^{c_{1}} T^{c_{2}}\right)^{(1+\varepsilon)(1-\alpha)}, \\
N^{*}(\alpha, T, Q) & \ll_{\varepsilon}\left(Q^{c_{3}} T^{c_{4}}\right)^{(1+\varepsilon)(1-\alpha)},
\end{aligned}
$$

with $c_{1}=c_{2}=3, c_{3}=5, c_{4}=3$. This was improved later by Motohashi [15] (for $4 / 5 \leq \alpha \leq 1)$ to $c_{1}=2, c_{2}=3, c_{3}=4, c_{4}=3$ and Jutila [12] to $c_{1}=c_{2}=2, c_{3}=4$, $c_{4}=2$ (for $4 / 5 \leq \alpha \leq 1$ ). Jutila mentioned that, according to a remark of Huxley, the value of $c_{3}$ can be improved to 3 if $\alpha$ is near to $1(1-c(\varepsilon) \leq \alpha \leq 1)$. This will be denoted by $c_{3}^{\prime}=3$. Wang [17] showed with this notation $c_{1}^{\prime}=c_{2}^{\prime}=3 / 2, c_{3}^{\prime}=3, c_{4}^{\prime}=3 / 2$.

In what follows we will use a method of S. W. Graham [7] and Heath-Brown [10] to improve this to $c_{3}^{\prime}=9 / 4$ and to obtain many new results. Our method will give estimates for $N(\alpha, T, \chi)$ with individual characters $\chi \bmod q$ as, for example,

$$
N\left(\alpha, T, \chi_{q}\right) \ll_{\varepsilon}(q T)^{(3 / 4+\varepsilon)(1-\alpha)} \quad\left(1-\varepsilon^{3} \leq \alpha \leq 1\right) .
$$

In particular, in case of $q=1$, we obtain results for the number of zeros $N(\alpha, T)$ of $\zeta(s)$.

In later applications we will often need density theorems for a given subset of all primitive characters with moduli $\leq Q$ instead of (2.7). Since the method applied in Section 3 yields much better results for these subsets, we will formulate our results in this more general setting. We will then obtain estimates of type $(2.6)-(2.8)$ as immediate consequences of the general theorem. In the following, let cond $\chi$ denote the conductor of $\chi$. 
THEOREM 1. Let $\mathcal{H}$ be a set of primitive characters $\chi$ with moduli $\leq M$ such that cond $\chi_{i} \bar{\chi}_{j} \leq K$ for any pair $\chi_{i}, \chi_{j}$ belonging to $\mathcal{H}$. Let $\mathcal{S}$ be a set of distinct pairs $\left(\chi_{j}, \varrho_{j}\right)$ with $L\left(\varrho_{j} \chi_{j}\right)=0$, where $\chi_{j} \in \mathcal{H}, \varrho_{j} \in R(\alpha, T)$. (The same character might naturally appear in $\mathcal{S}$ several times with different zeros.) Let $J$ denote the cardinality of $\mathcal{S}$ and let $\varepsilon$ be an arbitrary, sufficiently small positive number $\left(0<\varepsilon<c_{0}\right)$. Then for $\alpha>4 / 5$, $T \geq 3$, we have

$$
J \ll_{\varepsilon}\left(M^{1 /(2 \alpha-1)} K^{1 /((2 \alpha-1)(4 \alpha-3))} T^{2 /(4 \alpha-3)}\right)^{(3 / 4+\varepsilon)(1-\alpha)} .
$$

Corollary 1. The following estimates hold for $\alpha>4 / 5, T \geq 3$ :

$$
\begin{aligned}
N^{*}(\alpha, T, Q) & \ll_{\varepsilon}\left(Q^{(3+\varepsilon)(4 \alpha-1) /(4(4 \alpha-3)(2 \alpha-1))} T^{3+\varepsilon /(2(4 \alpha-3))}\right)^{(1-\alpha)}, \\
N(\alpha, T, q) & \ll_{\varepsilon}(q T)^{(3+\varepsilon)(1-\alpha) /(2(4 \alpha-3))}, \\
N\left(\alpha, T, \chi_{q}\right) & \ll_{\varepsilon}\left(q^{1 /(2 \alpha-1)} T^{2 /(4 \alpha-3)}\right)^{(3 / 4+\varepsilon)(1-\alpha)} \\
N(\alpha, T) & \ll_{\varepsilon} T^{(3+\varepsilon)(1-\alpha) /(2(4 \alpha-3))} .
\end{aligned}
$$

We remark that while the above estimate for $N^{*}(\alpha, T, Q)$ is distinctly sharper than that of Wang [17, the one for $N(\alpha, T, q)$ is just slightly better and for $\alpha \rightarrow 1$ asymptotically equal. Further, 2.10 is sharper than the density estimate in the $q$ and $T$ aspect for $\alpha>\frac{15}{16}$, and sharper in the $Q$ aspect for $\alpha>0.9020456 \ldots$. (The estimates of Wang [17] are better than the density theorem in case of $\alpha>23 / 24$ in all aspects.)

If at least one of $K$ and $M$ is small, the following result (Theorem 2 ) will be of interest.

THEOREM 2. Under the conditions of Theorem 1 for $\alpha>1-\varepsilon^{3}, T \geq 3$ we have

$$
\begin{aligned}
& J \ll_{\varepsilon}\left(K^{2}(M T)^{3 / 4}\right)^{(1+\varepsilon)(1-\alpha)}, \\
& J \ll_{\varepsilon}\left(M^{2}(K T)^{3 / 4}\right)^{(1+\varepsilon)(1-\alpha)}, \\
& J \ll_{\varepsilon}\left(M^{2} K^{2} T^{2 \varepsilon}\right)^{(1+\varepsilon)(1-\alpha)} .
\end{aligned}
$$

Corollary 2. For $\alpha>1-\varepsilon^{3}, T \geq 3$ we have

$$
\begin{aligned}
N\left(\alpha, T, \chi_{q}\right) & \ll_{\varepsilon}(q T)^{(3 / 4+\varepsilon)(1-\alpha)}, \\
N\left(\alpha, T, \chi_{q}\right) & \ll_{\varepsilon}\left(q^{2} T^{2 \varepsilon}\right)^{(1+\varepsilon)(1-\alpha)}, \\
N(\alpha, T, q) & \ll_{\varepsilon}\left(q^{4} T^{2 \varepsilon}\right)^{(1+\varepsilon)(1-\alpha)}, \\
N^{*}(\alpha, T, Q) & \ll_{\varepsilon}\left(Q^{6} T^{2 \varepsilon}\right)^{(1+\varepsilon)(1-\alpha)}, \\
N(\alpha, T) & \ll_{\varepsilon} T^{\varepsilon(1-\alpha)} .
\end{aligned}
$$

On the other hand, if $T$ is much smaller than $q$ or $Q$ (or it is bounded, for example, as in the proof of Linnik's theorem), then the following generalization of Heath-Brown's Lemma 11.1 [10] leads to improvements over Theorem 1 .

THEOREM 3. Suppose the conditions of Theorem 1 and let $\varphi=1 / 4$ if all characters in $\mathcal{H}$ have cube-free moduli (or order at most $\log M$ ), otherwise let $\varphi=1 / 3$. Then for $\alpha>1-\varepsilon^{2}, T \geq 3$ we have

$$
J \ll_{\varepsilon}\left((K M)^{2 \varphi+\varepsilon} T^{10 / \varepsilon}\right)^{(1-\alpha)} .
$$


Corollary 3. With the notation of Theorem 3 we have for $\alpha>1-\varepsilon^{2}, T \geq 3$

$$
\begin{aligned}
N^{*}(\alpha, T, Q) & \ll_{\varepsilon}\left(Q^{2+\varepsilon} T^{10 / \varepsilon}\right)^{1-\alpha}, \\
N(\alpha, T, q) & \ll_{\varepsilon} \begin{cases}\left(q^{1+\varepsilon} T^{10 / \varepsilon}\right)^{(1-\alpha)} & \text { if } q \text { is cube-free }, \\
\left(q^{4 / 3+\varepsilon} T^{10 / \varepsilon}\right)^{(1-\alpha)} & \text { otherwise } .\end{cases}
\end{aligned}
$$

If $K$ and $M$ are both significantly smaller than $T$, then the results $2.13,2.15-2.18$ are much better than (2.10), 2.11) and (2.12). The first results having an expression of the type $T^{o(1-\alpha)}$ for $\alpha \rightarrow 1$ were proved by Halász and Turán [8].

Finally, K. Ford [4] showed, as a consequence of his explicit estimate for the zetafunction (cf. (3.4) in our next section) the inequality

$$
N(\alpha, T) \ll T^{58.05(1-\alpha)^{3 / 2}} \log ^{15} T .
$$

In Section 4 we will deal with the other main ingredient of Linnik's theorem, the famous Deuring-Heilbronn phenomenon. This asserts that if an $L$-function has a Siegelzero, then other $L$-functions are free of zeros in some region.

Suppose that $\chi_{1}$ is a real primitive character $\bmod q_{1}$ such that $L\left(1-\delta_{1}, \chi_{1}\right)=0$ with a real $\delta_{1}$. Let $\chi_{2}$ be an arbitrary primitive character $\bmod q_{2}$ such that $L\left(1-\delta+i t, \chi_{2}\right)=0$, $\delta_{1}<\delta<1 / 6$. (The character $\chi_{2}$ may be equal to $\chi_{1}$.) Suppose $\varepsilon>0$ arbitrary, let $\left[q_{1}, q_{2}\right]$ denote the least common multiple of $q_{1}$ and $q_{2}$, and

$$
D=\left[q_{1}, q_{2}\right](|t|+1) \geq D_{0}(\varepsilon) .
$$

Jutila proved essentially the following version [12]:

$$
\delta_{1} \geq(1-6 \delta) D^{-(2+\varepsilon) \delta /(1-6 \delta)} / 8 \log D .
$$

Using Burgess' estimate, Graham [7] improved the exponent 2 to $3 / 2$ for bounded $t$. Later, using Heath-Brown's estimate (cf. (3.2) in the next section), Wang [17] showed essentially

$$
\delta_{1} \geq \frac{2}{3}(1-6 \delta) D^{-(3 / 2+\varepsilon) \delta /(1-6 \delta)} / \log D .
$$

As in the case of the density theorems, we need a more flexible form of this phenomenon in our application, where apart from the replacement of 2 by $3 / 2,\left[q_{1}, q_{2}\right]$ will be replaced by the quantity

$$
q_{1}^{1 / 2} q_{2}^{1 / 4}\left(\operatorname{cond} \chi_{1} \bar{\chi}_{2}\right)^{1 / 4} \leq\left[q_{1}, q_{2}\right]
$$

(This has no effect in Linnik's theorem, where all quantities can be equal to the same q.) Our version is as follows.

THEOREM 4. Let $\chi_{1}$ and $\chi_{2}$ be primitive characters $\bmod q_{1}$ and $q_{2}$, respectively, with $L\left(1-\delta_{1}, \chi_{1}\right)=L\left(1-\delta+i \gamma, \chi_{2}\right)=0, \chi_{1}, \delta_{1}$ real, $\delta_{1}<\delta<1 / 7$. Let $k$ be the conductor of $\chi_{1} \bar{\chi}_{2}$. Let $\varepsilon>0$ arbitrary,

$$
Y=\left(q_{1}^{2} q_{2} k(|\gamma|+2)^{2}\right)^{3 / 8} \geq Y_{0}(\varepsilon)
$$

sufficiently large. Then we have

$$
\delta_{1} \geq(1-\varepsilon)(1-6 \delta)(\log 2) \cdot Y^{-(1+\varepsilon) \delta /(1-6 \delta)} / \log Y .
$$


3. In the course of our proof we will use four different estimates for the $L$-functions associated to characters $\bmod q$. Let us define $\varphi=\varphi(\chi)=1 / 4$ if $q$ is cube-free, and let $\varphi=1 / 3$ otherwise. Let $k$ be any integer $\geq 3, \eta>0$ be a sufficiently small number. The first three estimates, to be used in Theorems 1,2 and 3 , respectively, are due to HeathBrown and make crucial use of Burgess' estimates for character sums. Let $s=\sigma+i t$, $\tau=|t|+2$. Then

$$
\begin{aligned}
L\left(\frac{1}{2}+i t, \chi\right) & \ll_{\eta}(q \tau)^{3 / 16+\eta}, & & \\
L(s, \chi) & \ll_{\eta}(q \tau)^{3 / 8(1-\sigma)+\eta} & & \text { if } 1 / 2 \leq \sigma \leq 1, \\
L(s, \chi) & \ll_{\eta, k} q^{(1-\sigma)(1+1 / k)+\eta} \tau & & \text { if } 1-1 / k \leq \sigma \leq 1 ;
\end{aligned}
$$

3.1) is contained in [9], 3.2) is a simple consequence of it by convexity, whereas (3.3) is Lemma 2.5 in $[10$.

The last estimate relies on the bound of Korobov-Vinogradov for which a sharper form is due to K. Ford [4]:

$$
\left|\zeta(\sigma+i t, u)-u^{-s}\right| \leq 76.2 t^{4.45(1-\sigma)^{3 / 2}} \log ^{2 / 3} t,
$$

for $0<u \leq 1, t \geq 3,1 / 2 \leq \sigma \leq 1$, where $\zeta(s, u)$ is Hurwitz' zeta-function. Since

$$
L(s, \chi)=\sum_{\ell=1}^{q} \frac{\chi(\ell)}{q^{\sigma+i t}} \zeta\left(\sigma+i t, \frac{\ell}{q}\right),
$$

the estimate (3.4) implies that for $t \geq 3,1 / 2 \leq \sigma \leq 1$,

$$
L(s, \chi) \ll q^{1-\sigma}\left(t^{4.45(1-\sigma)^{3 / 2}} \log ^{2 / 3} t+\log (q+1)\right) .
$$

Sometimes, e.g., in Theorem 2 we will apply a consequence of this, namely

$$
L(s, \chi) \ll\left(q \tau^{\eta}\right)^{1-\sigma} \log ((q+1) \tau) \quad \text { for } \sigma \geq 1-\eta^{2} / 20 .
$$

We will later make use of the fact that in view of 3.6 the following region is zero-free. Let $q \leq M, \chi$ primitive $\bmod q$. Then

$$
L(s, \chi) \neq 0 \quad \text { for } \quad \sigma \geq 1-\frac{c_{1}}{\max \left(\log M, \log ^{2 / 3} \tau \log _{2}^{1 / 3} \tau\right)}
$$

with the exception of at most one real zero belonging to a real primitive $\chi \bmod q \leq M$. This follows from the note after Satz 6.2 in Chapter VIII of Prachar's book [16], combined with Landau's theorem, in the form given in [2], $\$ 14$.

In the proof of Theorems 1 , 3 we will make use of Linnik's density lemma (see [16], p. 331).

LEMMA 1. The number of zeros of the function $L(s, \chi)(\chi(\bmod q))$ in the square

$$
\alpha \leq \sigma \leq 1, \quad|t-T| \leq(1-\alpha) / 2
$$

is

$$
\ll(1-\alpha) \log (q(|T|+2))+1 .
$$

In the proof of Theorem 4 we will use the following sharper form of Lemma 1 , a consequence of 3.6 .

LEMma 2. The number of zeros of $L(s, \chi)$ in 3.9 is

$$
\ll(1-\alpha) \log q+(1-\alpha)^{3 / 2} \log T+\log _{2}(q T) .
$$


Finally, Halász's inequality will play a central role in the proof.

LEMmA 3. Let $f(s, \chi)=\sum_{n=1}^{N} a_{n} \chi(n) n^{-s}$. Then

$$
\left(\sum_{j=1}^{J}\left|f\left(s_{j} \chi_{j}\right)\right|\right)^{2} \leq \sum_{n=1}^{N} \frac{\left|a_{n}\right|^{2}}{b_{n}} \sum_{j, k=1}^{J} \eta_{j} \bar{\eta}_{k} B\left(s_{j}+\bar{s}_{k}, \chi_{j} \bar{\chi}_{k}\right)
$$

where the $\eta_{j}$ are certain complex numbers of modulus 1 , and

$$
B(s, \chi)=\sum_{n=1}^{\infty} b_{n} \chi(n) n^{-s}
$$

where the $b_{n}$ are arbitrary non-negative numbers such that $b_{n}>0$ if $a_{n} \neq 0$, and $B(s, \chi)$ is absolutely convergent for all pairs $\left(s_{j}+\bar{s}_{k}, \chi_{j} \bar{\chi}_{k}\right)$.

This is a modified form of Halász's inequality given in [13], Lemma 1.7. For this form see Jutila [12, Lemma 7.

Clearly, we can suppose that $K \leq M^{2}$ in our proofs. Since there is at most one exception, the so-called Siegel zero to (3.8), we may suppose that the Siegel zero does not appear among our zeros. (The upper estimates for $J$ are at least a positive constant in Theorems 1 3.) Further, it is enough to show our theorems for non-principal characters and then, additionally, for just the zeta-function. Thus, we will first show Theorems 13 for non-principal characters, and we will then mention the slight modifications which prove them for the zeta-function.

Instead of using pseudocharacters, we will use Graham's approach [7], in the way Heath-Brown did in [10], Lemma 11.1.

In the proof, $\varepsilon$ will denote a sufficiently small positive constant, not necessarily the same as in the formulation of the theorems.

We will use parameters

$$
W=e^{w}, \quad U=e^{\mathcal{L}}, \quad V=U W=e^{\mathcal{L}+w}=e^{v}, \quad X=e^{x}
$$

to be specified later, with the property

$$
\mathcal{L} \ll_{\varepsilon} w<\mathcal{L}<x \ll_{\varepsilon} \mathcal{L} .
$$

Following [10], let us define Graham's weights

$$
\psi_{d}= \begin{cases}\mu(d) & \text { for } 1 \leq d \leq U, \\ \mu(d) \frac{\log (V / d)}{\log (V / U)} & \text { for } U \leq d \leq V, \\ 0 & \text { for } d \geq V\end{cases}
$$

and a special case of this $(U=1)$, namely

$$
\theta_{d}= \begin{cases}\mu(d) \frac{\log (W / d)}{\log W} & \text { for } 1 \leq d \leq W \\ 0 & \text { for } d \geq W\end{cases}
$$

We set

$$
\begin{gathered}
\Psi(n)=\sum_{d \mid n} \psi_{d}, \quad \vartheta(n)=\sum_{d \mid n} \theta_{d}, \\
\alpha=1-\delta,
\end{gathered}
$$


and we will choose our parameters in such a way that

$$
x \gg \log M+\log ^{2 / 3} T \log _{2}^{1 / 3} T
$$

should be satisfied. In this way, by (3.8), we will have, with the exception of the Siegel zero, for any relevant $\varrho_{\nu}=\beta_{\nu}+i \gamma_{\nu}$

$$
\delta \geq 1-\beta_{\nu}=\delta_{\nu} \gg x^{-1} .
$$

We take $\chi=\chi_{k} \neq \chi_{0}$ with conductor $q=q_{k} \neq 1$, and with a zero $\varrho_{k}=\beta_{k}+i \gamma_{k}=\varrho=$ $\beta+i \gamma$ of $L(s, \chi)$ and

$$
S(X)=\sum_{n=1}^{\infty} \Psi(n) \vartheta(n) \chi(n) n^{-\varrho} e^{-n / X}=\frac{1}{2 \pi i} \int_{(1)} L(s+\varrho, \chi) \Gamma(s) X^{s} F(s+\varrho) d s,
$$

where

$$
F(s)=\sum_{i \leq V, j \leq W} \psi_{i} \theta_{j} \chi([i, j])[i, j]^{-s} .
$$

([i,j] always denotes the least common multiple of $i$ and $j$.)

We move the line of integration to $\sigma=1-\beta-h$, where $h$ will be chosen later with $h<1-\beta$. (The integrand is regular between $\sigma=1$ and $\sigma=1-\beta-h$.) Using the estimates $\Gamma(s) \ll e^{-|t|}$ and

$$
F(s+\varrho) \ll \sum_{i \leq V, j \leq W}[i, j]^{-1+h} \ll \sum_{n \leq V W} d^{2}(n) n^{-1+h} \ll(V W)^{h} \mathcal{L}^{3},
$$

by $3.1-3.2$ we obtain

$$
\begin{aligned}
S(X) & =\int_{(1-\beta-h)} L(s+\varrho, \chi) F(s+\varrho) \Gamma(s) X^{s} d s \\
& \ll_{\varepsilon}\left((M T)^{3 / 8} V W X^{-1}\right)^{h}(U M T)^{\varepsilon^{2}} X^{1-\beta} .
\end{aligned}
$$

If

$$
X \gg_{\varepsilon}\left((M T)^{3 / 8} V W\right)^{1 /(1-\delta / h)}(U M T)^{2 \varepsilon^{2} /(h(1-\delta / h))},
$$

then

$$
S(X)=O\left(\mathcal{L}^{-1}\right)
$$

where (here and later) the constants implied by the $O$ symbols may depend on $\varepsilon$.

Taking into account $\Psi(n)=0$ for $2 \leq n \leq U$, we have

$$
S\left(U / \mathcal{L}^{2}\right)=e^{-\mathcal{L}^{2} / U}+O\left(\sum_{n>U} d(n) e^{-n \mathcal{L}^{2} / U}\right)=1+O(1 / \mathcal{L}) .
$$

Thus 3.27 implies, under the condition 3.26 for $X$,

$$
\sum_{n=1}^{\infty} \Psi(n) \vartheta(n) \chi(n) n^{-\varrho}\left(e^{-n / X}-e^{-n \mathcal{L}^{2} / U}\right)=1+O\left(\mathcal{L}^{-1}\right)
$$

Now we will use Halász's inequality in the form 3.12 with

$$
\begin{aligned}
& a_{n}=\Psi(n) \vartheta(n) n^{-1 / 2}\left(e^{-n / X}-e^{-n \mathcal{L}^{2} / U}\right), \\
& b_{n}=\vartheta^{2}(n)\left(e^{-n / X}-e^{-n \mathcal{L}^{2} / U}\right), \quad s_{j}=\varrho_{j}-1 / 2 .
\end{aligned}
$$


Using the estimate of Graham [6, p. 84] that

$$
\sum_{1<n \leq N} \Psi^{2}(n)= \begin{cases}0 & \text { for } 1 \leq N \leq U, \\ \frac{N \log (N / U)}{\log ^{2}(V / U)}+O\left(\frac{N}{\log ^{2}(V / U)}\right) & \text { for } U \leq N \leq V, \\ \frac{N}{\log (V / U)}+O\left(\frac{N}{\log ^{2}(V / U)}\right) & \text { for } \quad N \geq V,\end{cases}
$$

by partial summation we obtain (cf. [10, (11.14)) for $x>v$

$$
\sum_{n=1}^{\infty} \frac{\left|a_{n}\right|^{2}}{b_{n}}=\sum_{n=1}^{\infty} \frac{\Psi^{2}(n)}{n}\left(e^{-n / X}-e^{n \mathcal{L}^{2} / U}\right)=\left(1+O\left(\mathcal{L}^{-1}\right)\right) \frac{2 x-\mathcal{L}-v}{2(v-\mathcal{L})} .
$$

Any term with $\chi_{j} \bar{\chi}_{k} \neq \chi_{0}$ on the right-hand side of 3.12 will be, similarly to 3.25 (3.27,

$$
\begin{aligned}
B\left(s_{j}+\bar{s}_{k}, \chi_{j} \bar{\chi}_{k}\right) & =\sum_{n=1}^{\infty} \vartheta^{2}(n) \chi_{j} \bar{\chi}_{k}(n)=n^{-\left(\varrho_{j}+\bar{\varrho}_{k}-1\right)}\left(e^{-n / x}-e^{-n \mathcal{L}^{2} / U}\right) \\
& \ll_{\varepsilon}\left((K T)^{3 / 8} W^{2} U^{-1}\right)^{h} \cdot(K T)^{\varepsilon^{2}} \mathcal{L}^{3} \cdot U^{2 \delta} \ll \mathcal{L}^{-1}
\end{aligned}
$$

if

$$
U \gg_{\varepsilon}\left((K T)^{3 / 8} W^{2}\right)^{1 /(1-2 \delta / h)} \cdot(W K T)^{2 \varepsilon^{2} /(h(1-2 \delta / h))} .
$$

Let us consider the case $\chi_{j} \bar{\chi}_{k}=\chi_{0, q}=\chi_{0}$ now. Then, in the case of (3.34), we have, similarly,

$$
\begin{aligned}
B\left(s_{j}\right. & \left.+\bar{s}_{k}, \chi_{0}\right)=\sum_{n=1}^{\infty} \vartheta^{2}(n) \chi_{0}(n) n^{1-\varrho_{j}-\bar{\varrho}_{k}}\left(e^{-n / X}-e^{-n \mathcal{L}^{2} / U}\right) \\
& =\frac{1}{2 \pi i} \int_{(1)} L\left(s+\varrho_{j}+\bar{\varrho}_{k}-1, \chi_{0}\right) G_{q}\left(s+\varrho_{j}+\bar{\varrho}_{k}-1\right) \Gamma(s)\left(X^{s}-\left(\frac{U}{\mathcal{L}^{2}}\right)^{s}\right) d s \\
& =\frac{\varphi(q)}{q} G_{q}(1) \Gamma\left(2-\varrho_{j}-\bar{\varrho}_{k}\right)\left(X^{2-\varrho_{j}-\bar{\varrho}_{k}}-\left(\frac{U}{\mathcal{L}^{2}}\right)^{2-\varrho_{j}-\bar{\varrho}_{k}}\right)+O\left(\mathcal{L}^{-1}\right),
\end{aligned}
$$

where

$$
G_{q}(s)=\sum_{\substack{j \leq W, k \leq W \\(j, q)=(k, q)=1}} \theta_{j} \theta_{k}[j, k]^{-s}
$$

The following proposition can easily be proved.

Proposition. We have

$$
\frac{\varphi(q)}{q} G_{q}(1) \leq \frac{1+C / w}{w} \quad(w=\log W) .
$$

Proof. We will investigate the finite Dirichlet polynomial $G_{q}(s)$ for real $s>1, s \rightarrow 1$. We have

$$
E(s)=G_{q}(s) L\left(s, \chi_{0}\right)=\sum_{\substack{n=1 \\(n, q)=1}}^{\infty} \frac{\vartheta^{2}(n)}{n^{s}} \leq \sum_{n=1}^{\infty} \frac{\vartheta^{2}(n)}{n^{s}} \leq\left(1+\frac{C}{w}\right) w^{-1} \zeta(s)
$$


since, applying (3.31) in the special case of $\Psi=\vartheta$ (that is $U=1, V=W$ ), we have for all $y \geq 1$

$$
\sum_{1<n \leq y} \vartheta^{2}(n) \leq \frac{y(1+C / w)}{w}-1 .
$$

Taking the limit $s \rightarrow 1^{+}$in 3.37), we obtain the Proposition.

Fix a pair $\left(\chi_{j}, \varrho_{j}\right)(1 \leq j \leq J)$, and consider all zeros $\varrho_{k_{\nu}}\left(1 \leq \nu \leq J^{\prime}\right)$ belonging to the same $L\left(s, \chi_{j}\right)$ (including $\varrho_{j}$ itself). Let $\varrho_{j}=\beta_{j}+i \gamma_{j}=1-\delta_{j}+i \gamma_{j}, \varrho_{k}=\beta_{k}+i \gamma_{k}=$ $1-\delta_{k}+i \gamma_{k}$. According to 3.35 and the Proposition, we have (by $\chi_{j}=\chi_{k}$ )

$$
\begin{aligned}
B\left(s_{j}+\bar{s}_{k}, \chi_{j} \bar{\chi}_{k}\right) \ll \frac{X^{\delta_{j}+\delta_{k}}}{w\left|\delta_{j}+\delta_{k}+i\left(\gamma_{j}-\gamma_{k}\right)\right|} \\
\qquad \begin{cases}\frac{X^{2 \delta}}{w \cdot \delta} & \text { if }\left|\gamma_{j}-\gamma_{k}\right| \leq \delta, \\
\frac{X^{2 \delta}}{w \cdot n \delta} & \text { if } n \delta \leq\left|\gamma_{j}-\gamma_{k}\right| \leq(n+1) \delta,\end{cases}
\end{aligned}
$$

using (3.21) and the fact that $y^{-1} e^{y}$ is increasing for $y \geq 1$. Using Lemma 1 we see that the number of possible zeros $\varrho_{k}$ of $L(s, \chi)$ with $n \delta \leq\left|\gamma_{j}-\gamma_{k}\right| \leq(n+1) \delta$ and $\delta_{k} \leq \delta$ is

$$
\ll \delta \log (M(T+n))+1 .
$$

Now, these imply for any fixed $j$

$$
\sum_{\nu=1}^{J^{\prime}}\left|B\left(s_{j}+\bar{s}_{k_{\nu}}, \chi_{0}\right)\right| \ll \frac{X^{2 \delta} \delta \log \left(M\left(T+J^{\prime}\right)\right)+1}{w \delta} \log J^{\prime}
$$

and so we have in Halász's Lemma (Lemma 3, by 3.8, 3.32 and 3.41

$$
J^{2} \ll \frac{x}{v-\mathcal{L}}\left(J \frac{X^{2 \delta} \log (M T)}{w} \log ^{2} J+J^{2} \mathcal{L}^{-1}\right) .
$$

Hence by $3.14-3.15$

$$
J \ll_{\varepsilon} X^{2 \delta(1+\varepsilon)}
$$

if

$$
w \gg_{\varepsilon} \log M T \text {. }
$$

In order to prove Theorem 1, we may choose

$$
\begin{gathered}
h=1 / 2, \quad W=(M T)^{\varepsilon}, \quad U=(K T)^{(3 / 8+\varepsilon) /(1-4 \delta)}(M T)^{10 \varepsilon}, \\
X=(M T)^{(3 / 8+50 \varepsilon) /(1-2 \delta)}(K T)^{(3 / 8+50 \varepsilon) /((1-2 \delta)(1-4 \delta))} .
\end{gathered}
$$

Then all conditions $3.15,3.20,3.26$, 3.34, 3.44 are satisfied, and this proves (3.43), that is, Theorem 1

For the proof of Theorem 2 2.11, we choose

$$
h=\varepsilon, \quad W=(M T)^{\varepsilon}, \quad U=K(M T)^{10 \varepsilon}, \quad X=K(M T)^{3 / 8+100 \varepsilon},
$$

but instead of 3.2 , we will use the estimate 3.7 for the $L$-functions in the estimate of the $B$-functions. Accordingly, instead of 3.33 , we have now

$$
B\left(s_{j}+s_{k}, \chi_{j} \bar{\chi}_{k}\right) \ll\left(K T^{\varepsilon} W^{2} U^{-1}\right)^{h} \mathcal{L}^{3} U^{2 \delta} \cdot(K T)^{\varepsilon^{2}} \ll \mathcal{L}^{-1} .
$$

Again, all conditions 3.15), 3.20, 3.26, 3.44) are satisfied and thus 2.11) is proved. 
The proof of 2.12 runs completely analogously. In this case the role of $K$ and $M$ is 'interchanged'. We choose

$$
h=\varepsilon, \quad W=(M T)^{\varepsilon}, \quad U=(K T)^{50 \varepsilon} M^{10 \varepsilon}, \quad X=M^{1+50 \varepsilon}(K T)^{3 / 8+100 \varepsilon}
$$

and use the estimate 3.7$)$ in the evaluation of $S(X)$ in $3.23-(3.25)$ while we use 3.2 in the estimate of the $B$-functions as in $3.33-3.34$.

Finally, in case of 2.13 we will use the estimate 3.7$)$ both in the evaluation of $S(X)$ and in the estimation of the $B$-function. Accordingly, we choose in this case

$$
h=\varepsilon, \quad W=(M T)^{\varepsilon / 4}, \quad U=K^{1+\varepsilon}(M T)^{\varepsilon}, \quad X=(K M)^{1+\varepsilon} T^{2 \varepsilon} .
$$

To prove Theorem 3 we can choose

$$
h=\varepsilon, \quad W=(M T)^{\varepsilon}, \quad U=K^{\varphi+\varepsilon} M^{3 \varepsilon} T^{2 / \varepsilon}, \quad X=(K M)^{\varphi+10 \varepsilon} T^{10 / \varepsilon} .
$$

Applying the estimate (3.3) with $k=\left[\varepsilon^{-1}\right]$ and $\eta=\varepsilon^{2} / 2$, we obtain, instead of (3.25) and (3.33), the estimates

$$
S(X) \ll\left(M^{(1+2 \varepsilon) \varphi} T^{1 / \varepsilon} V W X^{-1}\right)^{\varepsilon}(U M T)^{\varepsilon^{2}} X^{1-\beta} \ll \mathcal{L}^{-1}
$$

and

$$
B\left(s_{j}+\bar{s}_{k}, \chi_{j} \bar{\chi}_{k}\right) \ll\left(K^{(1+2 \varepsilon) \varphi} T^{1 / \varepsilon} W^{2} U^{-1}\right)^{\varepsilon}(U K T)^{\varepsilon^{2}} U^{2 \delta} \ll \mathcal{L}^{-1} .
$$

Since the conditions 3.15, 3.20, 3.44 are again satisfied, Theorem 3 is also proved.

In the case of the Riemann zeta-function, Theorem 3 is clearly much weaker than any of Theorems 1 and 2 Theorem 2 clearly follows from 2.21 for the zeta-function.

On the other hand, in case of the zeta-function, Theorem 1 (that is 2.9 ) follows for $\alpha \geq 4 / 5$ from Theorem 11.4 of Ivić [11] since

$$
\frac{3}{2 \alpha}<\frac{3}{2(4 \alpha-3)}
$$

4. Proof of Theorem 4. Following Jutila [12], we will use the idea of Selberg [14] to apply pseudocharacters

$$
f_{r}(n)=f((r, n))
$$

with multiplicative arithmetic functions $f$ where $(a, b)$ denotes the greatest common divisor of $a$ and $b$.

Let us use the abbreviation

$$
f_{r} f_{r^{\prime}}(n)=f_{r}(n) f_{r^{\prime}}(n) .
$$

For the exceptional real non-principal character $\chi_{1}$ let

$$
a_{n}=\sum_{d \mid n} \chi_{1}(d)=\prod_{p^{\alpha} \| n}\left(1+\chi_{1}(p)+\ldots+\chi_{1}^{\alpha}(p)\right) \geq 0 .
$$

If $n$ is square-free, then $a_{n}=0$ if there exists a prime divisor $p$ of $n$ with $\chi_{1}(p)=-1$. If $n$ is square-free and $\chi_{1}(p)=1$ for all $p \mid n$, then $a_{n}=2^{\omega(n)}$, where $\omega(n)$ is the number of prime factors of the square-free number $n\left(a_{1}=1\right)$.

Both $\chi_{1}(n)$ and $\chi_{2}(n)$ can be considered as characters $\bmod q=\left[q_{1}, q_{2}\right]$. Let $\chi_{0}$ be the principal character $\bmod q, \mu(n)$ the Möbius function. Let $\sum^{\prime}$ denote summation over all square-free numbers coprime to $q$. Let $S=\sum_{r \leq R}^{\prime} a_{r} r^{-1}$ with the parameter $R$ to be chosen later. 
In the course of proof we will need the following lemmas:

LEMMA 4. Let $\chi$ be a Dirichlet character, $f$ a multiplicative function, $r$ and $r^{\prime}$ square-free numbers such that $\chi_{1}(p)=1$ for all prime divisors of $r r^{\prime}$, and define for $\sigma>1$

$$
G_{r, r^{\prime}}(s, \chi)=\sum_{n=1}^{\infty} \mu^{2}(n) a_{n} \chi(n) f_{r} f_{r^{\prime}}(n) n^{-s}
$$

Then

$$
G_{r, r^{\prime}}(s, \chi)=L(s, \chi) L\left(s, \chi \chi_{1}\right) P_{r, r^{\prime}}(s, \chi) Q(s, \chi)
$$

where

$$
\begin{aligned}
P_{r, r^{\prime}}(s, \chi) & =\prod_{\substack{p \mid r r^{\prime} \\
p \nmid\left(r, r^{\prime}\right)}}\left(1+\frac{2 \chi(p) f(p)}{p^{s}}\right) \prod_{p \mid\left(r, r^{\prime}\right)}\left(1+\frac{2 \chi(p) f^{2}(p)}{p^{s}}\right) \prod_{p \mid r r^{\prime}}\left(1+\frac{2 \chi(p)}{p^{s}}\right)^{-1}, \\
Q(s, \chi) & =\prod_{\chi_{1}(p)=1}\left(1-\frac{\chi(p)}{p^{s}}\right)^{2}\left(1+2 \frac{\chi(p)}{p^{s}}\right) \prod_{\chi_{1}(p)=-1}\left(1-\frac{\chi^{2}(p)}{p^{2 s}}\right) .
\end{aligned}
$$

Proof. This is Lemma 9 of Jutila [12].

LEMMA 5. In the preceding lemma, choose

$$
f(n)=\mu(n) 2^{-\omega(n)} n,
$$

and suppose also that $L\left(\chi_{1}, \beta_{1}\right)=0$, where $\beta_{1}=1-\delta_{1}$ is a real number satisfying $3 / 4<\beta_{1}<1$. Then for the sum

$$
T=\sum_{n=1}^{\infty} a_{n} e^{-n / Y} n^{-\beta_{1}}\left(\sum_{r \leq R}^{\prime} a_{r} f_{r}(n) r^{-1}\right)^{2}
$$

we have for every $R$ the asymptotic formula

$$
T=\frac{\varphi(q)}{q} Q\left(1, \chi_{0}\right) L\left(1, \chi_{1}\right) \Gamma\left(\delta_{1}\right) Y^{\delta_{1}} S+O_{\varepsilon}\left(R q_{1}^{3 / 16+\varepsilon} Y^{1 / 2-\beta_{1}+\varepsilon}\right) .
$$

Proof. This is a sharpened form of Lemma 10 of [12], with the only change that on the line $\sigma=1 / 2$ we use the estimate (3.1) of Heath-Brown for $L\left(s, \chi_{1}\right)$. This form of Lemma 5 appears also as Lemma 12 of [17]. Therefore we can replace $q^{1 / 4}$ of [12] by $q_{1}^{3 / 16}$.

Lemma 6. For every $R_{0}$ there exists an $R \in\left[R_{0}, 2 R_{0}\right]$ such that

$$
S=S(R) \geq \frac{\varphi(q)}{q} Q\left(1, \chi_{0}\right) \mathcal{L}\left(1, \chi_{1}\right) \delta_{1}^{-1}+O_{\varepsilon}\left(R^{-1 / 2+\varepsilon} q_{1}^{3 / 16+\varepsilon}\right) .
$$

Proof. This is also a sharpened form of Lemma 11 of [12, at least for the suitably chosen $R$. Here we must further modify the proof. The generating function of $\mu^{2}(n) a_{n} \chi_{0}(n)$ is $F(s)=L\left(s, \chi_{1}\right) L\left(s, \chi_{0}\right) Q\left(s, \chi_{0}\right)$.

Hence we have for all $R$

$$
S \geq R^{-\delta_{1}} \sum_{r \leq R}^{\prime} \frac{a_{r}}{r^{\beta_{1}}}=\frac{R^{-\delta_{1}}}{2 \pi i} \int_{a-i R q}^{a+i R q} \frac{F\left(s+\beta_{1}\right) R^{s}}{s} d s+O\left(R^{-1 / 2}\right),
$$


where $a=\delta_{1}+1 / \log (q R)$. Moving the line of integration to the line $\operatorname{Re}\left(s+\beta_{1}\right)=1 / 2+\varepsilon$, we get a pole at $s=\delta_{1}$ with residue

$$
R^{-\delta_{1}} \cdot \frac{R^{\delta_{1}}}{\delta_{1}} \cdot Q\left(1, \chi_{0}\right) \frac{\varphi(q)}{q} L\left(1, \chi_{1}\right)
$$

and

$$
\frac{1}{2 \pi i} \int_{\delta_{1}-1 / 2+\varepsilon-i R q}^{\delta_{1}-1 / 2+\varepsilon+i R q} \frac{F\left(s+\beta_{1}\right) R^{s-\delta_{1}}}{s} d s+O\left(R^{-1 / 2}\right)=I(R)+O\left(R^{-1 / 2}\right) .
$$

The average of the real integral $I(R)$ is clearly

$$
E\left(R_{0}\right)=\frac{1}{R_{0}} \int_{R_{0}}^{2 R_{0}} I(R) d R=\frac{1}{2 \pi i} \int_{\delta_{1}-1 / 2+\varepsilon-i R q}^{\delta_{1}-1 / 2+\varepsilon+i R q} \frac{F\left(s+\beta_{1}\right) R_{0}^{s-\delta_{1}}}{s\left(s+\beta_{1}\right)}\left(2^{s+\beta_{1}}-1\right) d s .
$$

Using again the estimate (3.1) of Heath-Brown for $L\left(s, \chi_{1}\right)$, we obtain for $E\left(R_{0}\right)$ the estimate given in the error term of 4.9 .

LEMma 7. Let $\beta_{1}$ be as in the preceding lemmas, and suppose also that $L(\varrho, \chi)=0$, where $\chi$ is a character $\left(\bmod q_{2}\right)$, and $\varrho=\beta+i \gamma, 3 / 4<\beta<\beta_{1}$. Put $D=\left(q_{2} k(|\gamma|+2)^{2}\right)^{3 / 8}$. Then in the case $\chi \neq \chi_{0}, \chi_{1}$ we have, for the quantity $T$ defined by 4.7), the estimates

$$
T \geq S^{2}\left(1+Y^{(1+\varepsilon)\left(\beta-\beta_{1}\right)}\right)+O_{\varepsilon}\left(R D^{1 / 2+\varepsilon} Y^{1 / 2-\beta_{1}+\varepsilon}\right)
$$

for all $R$. If $\chi=\chi_{0}$ or $\chi_{1}$, then for every $R_{0}$ there exists an $R \in\left[R_{0}, 2 R_{0}\right]$ such that either

$$
T \geq S^{2}\left(1+(1-\varepsilon) Y^{(1+\varepsilon)\left(\beta-\beta_{1}\right)}\right)+O_{\varepsilon}\left(R_{0} D^{1 / 2+\varepsilon} Y^{1 / 2-\beta_{1}+\varepsilon}\right)
$$

or

$$
\delta_{1} \geq \varepsilon Y^{\beta-1}|\Gamma(1-\varrho)|^{-1}\left\{1+O_{\varepsilon}\left(R_{0}^{-1 / 2+\varepsilon} q_{1}^{3 / 16+\varepsilon}\right)\right\} .
$$

Proof. This is again a sharpened form of Lemma 12 of [12] which can be proved using the estimate (3.1) and our Lemma 6 in place of Lemma 11 of [12].

Another minor change in the proof is that in (5.7)-(5.8) of [12] we will replace the factor $1 / 2$ by $1-\varepsilon$, and, accordingly, for the $\theta$ in (5.9) of [12] we have the inequality $(2-\varepsilon)^{-1}<\theta<\varepsilon^{-1}$ in place of $2 / 3<\theta<2$. This makes 4.15 slightly stronger and the less crucial 4.16) weaker.

We remark that in the formula before (5.7) of [12] on the right side of the inequality a factor $S$ is missing from $(\varphi(q) / q) Q\left(1, \chi_{0}\right) L\left(1, \chi_{1}\right)|\Gamma(1-\varrho)| Y^{1-\beta}$ by a misprint (see the corresponding formula for $T_{\chi}$ above it).

Proof of Theorem 4. The proof follows that of Jutila [12], with slight changes, so we will be brief. For all $R_{0}$ we can choose a fixed value of $R$ such that Lemmas 6 and 7 should hold with the same $R$. Since $R \in\left[R_{0}, 2 R_{0}\right]$ it is irrelevant whether we write $R$ or $R_{0}$ in the error terms. We can suppose $\delta_{1} \ll(\log Y)^{-1}$ and $\delta_{1} \ll\left(\log q_{1}\right)^{-1}$, otherwise (2.27) holds. The choices of $R_{0}$ and $Y$ will imply $\log \left(R_{0} Y\right) \ll \log D$ due to $q_{1} \leq q_{2} k$. Let us consider first the case $\chi \neq \chi_{0}, \chi_{1}$. Then the comparison of Lemmas 5 and 7 namely (4.8) and (4.14), imply, with the notation

$$
B=\frac{\varphi(q)}{q} Q\left(1, \chi_{0}\right) \mathcal{L}\left(1, \chi_{1}\right)
$$


the inequality

$$
\begin{aligned}
B \Gamma\left(\delta_{1}\right) Y^{\delta_{1}} S \geq & S^{2}\left(1+Y^{(1+\varepsilon)\left(\beta-\beta_{1}\right)}\right) \\
& +O_{\varepsilon}\left(R_{0} q_{1}^{3 / 16+\varepsilon} Y^{1 / 2-\beta_{1}+\varepsilon}\right)+O_{\varepsilon}\left(R_{0} D^{1 / 2+\varepsilon} Y^{1 / 2-\beta_{1}+\varepsilon}\right) .
\end{aligned}
$$

The first error term can be neglected, since it is inferior to the second, in view of $D>$ $\left(q_{2} k\right)^{3 / 8} \geq q_{1}^{3 / 8}$. Cancelling this inequality and replacing $S$ by the estimate from Lemma 6 . by $S \geq a_{1}=1$ we obtain

$$
\begin{aligned}
B \Gamma\left(\delta_{1}\right) Y^{\delta_{1}} \geq & B \delta_{1}^{-1}\left(1+Y^{(1+\varepsilon)\left(\beta-\beta_{1}\right)}\right) \\
& +O_{\varepsilon}\left(R_{0} D^{1 / 2+\varepsilon} Y^{1 / 2-\beta_{1}+\varepsilon}\right)+O_{\varepsilon}\left(R_{0}^{-1 / 2+\varepsilon} q_{1}^{3 / 16+\varepsilon}\right) .
\end{aligned}
$$

Here $\Gamma\left(\delta_{1}\right) \delta_{1}=1+O\left(\delta_{1}\right)$, and from the integral representation 4.10 we obtain an $R \in\left[q_{1}, 2 q_{1}\right]$ such that by $\delta_{1} \ll\left(\log q_{1}\right)^{-1}$,

$$
1 \ll R^{-\delta_{1}} \sum_{n \leq R} \frac{a_{r}}{r}=B \delta_{1}^{-1}+O\left(q_{1}^{-1 / 2}\right)+O\left(q_{1}^{-1 / 16+2 \varepsilon}\right) .
$$

Thus we may divide by $B \Gamma\left(\delta_{1}\right) \gg 1$ to obtain

$$
\begin{aligned}
Y^{\delta_{1}} \geq & 1+Y^{-(1+\varepsilon) \delta}+O\left(\delta_{1}\right)+O_{\varepsilon}\left(R_{0} D^{1 / 2+\varepsilon} Y^{1 / 2-\beta_{1}+\varepsilon}\right) \\
& +O_{\varepsilon}\left(R_{0}^{-1 / 2+\varepsilon} q_{1}^{3 / 16+\varepsilon}\right) .
\end{aligned}
$$

Now we will choose $R_{0}$ and $Y$ in such a way that the last two error terms on the right side should be of lower order of magnitude than $Y^{-(1+\varepsilon) \delta}$. Let

$$
Y=\left(D q_{1}^{3 / 4}\right)^{\frac{1}{1-6 \delta}}+\varepsilon_{1}, \quad R_{0}=q_{1}^{3 / 8+\varepsilon_{2}}\left(D q_{1}^{3 / 4}\right)^{\frac{2 \delta}{1-6 \delta}}+\varepsilon_{2},
$$

where $\varepsilon_{1}$ and $\varepsilon_{2}$ are properly chosen small numbers depending on $\varepsilon$. (That is, $\varepsilon_{1}(\varepsilon) \rightarrow 0$ and $\varepsilon_{2}(\varepsilon) \rightarrow 0$ as $\varepsilon \rightarrow 0$.) With the above choice we obtain from 4.21 the estimate

$$
e^{\delta_{1} \log Y}-1+O\left(\delta_{1}\right) \geq(1-\varepsilon) Y^{-(1+\varepsilon) \delta} .
$$

Now, if $u=\delta_{1} \log Y \geq \log 2$, then 2.27) clearly holds. If $u<\log 2$, then $e^{u}-1 \leq u / \log 2$ and so 4.23 yields

$$
\delta_{1} \log Y / \log 2 \geq(1-2 \varepsilon) Y^{-(1+\varepsilon) \delta},
$$

which proves Theorem 4 when $\chi \neq \chi_{0}, \chi_{1}$.

If $\chi=\chi_{0}$ or $\chi=\chi_{1}$ and 4.15 holds, then the same argument as above applies and we obtain 4.24 with $1-2 \varepsilon$ replaced by $1-3 \varepsilon$.

If $\chi=\chi_{0}$ or $\chi=\chi_{1}$ and 4.16 holds, then (4.16) implies the estimate

$$
\delta_{1}>\varepsilon(1-\varepsilon) Y^{-\delta}\left|\frac{\Gamma(1-\varrho)^{-1}}{1-\varrho}\right||1-\varrho|,
$$

which clearly proves our theorem if

$$
|1-\varrho|>\varepsilon^{-1} / \log Y \text {. }
$$

This is trivially true for $\chi_{0}$. If $\chi=\chi_{1}$, then $Y \geq q_{1}^{9 / 8}(|t|+1)^{3 / 4}>q_{1}$. If

$$
|1-\varrho| \leq \varepsilon^{-1} / \log Y
$$

then we can apply Lemma 8.4 of Heath-Brown [10]. This asserts, with our notation, that

$$
\frac{1}{\delta_{1} \log Y} \leq \frac{1}{\delta_{1} \log q_{1}} \leq e^{(2 / 3) \delta \log q_{1}}=q_{1}^{2 \delta / 3} \leq Y^{2 \delta / 3}
$$

if $q_{1}>q_{0}(\varepsilon)$, and therefore immediately proves (2.27). 
If $q_{1} \leq q_{0}(\varepsilon)$, then all zeros of any $L$-functions $\bmod q \leq q_{0}(\varepsilon)$ are at a distance at least $d_{0}(\varepsilon)$ from 1 . Therefore 4.26 will be true if

$$
\log Y>\left(\varepsilon d_{0}(\varepsilon)\right)^{-1},
$$

i.e. when $Y>Y_{0}(\varepsilon):=e^{\left(\varepsilon d_{0}(\varepsilon)\right)^{-1}}$.

Acknowledgments. This research was partially supported by ERC-AdG. 321104 and National Research Development and Innovation Office, NKFIH, K 119528.

\section{References}

[1] F. Carlson, Über die Nullstellen der Dirichletschen Reihen und der Riemannscher $\zeta$-Funktion, Arkiv. F. Math. Astr. Fys. 15 (1920), No. 20.

[2] H. Davenport, Multiplicative Number Theory, 2nd ed., Grad. Texts in Math. 74, Springer, New York, 1980.

[3] E. Fogels, On the zeros of L-functions, Acta Arith. 11 (1965), 67-96.

[4] K. Ford, Zero-free regions for the Riemann zeta function, in: Number Theory for the Millennium, II (Urbana, IL, 2000), A. K. Peters, Natick, MA, 2002, 25-56.

[5] P. X. Gallagher, A large sieve density estimate near $\sigma=1$, Invent. Math. 11 (1970), 329-339.

[6] S. W. Graham, Application of sieve methods, Ph.D. Thesis, Univ. of Michigan, 1977.

[7] S. W. Graham, On Linnik's constant, Acta Arith. 39 (1981), 163-179.

[8] G. Halász, P. Turán, On the distribution of roots of Riemann zeta and allied functions, I, J. Number Theory 1 (1969), 121-137.

[9] D. R. Heath-Brown, Hybrid bounds for Dirichlet L-functions. II, Quart. J. Math. Oxford Ser. (2) 31 (1980), 157-167.

[10] D. R. Heath-Brown, Zero-free regions for Dirichlet L-functions, and the least prime in an arithmetic progression, Proc. London Math. Soc. (3) 64 (1992), 265-338.

[11] A. Ivić, The Riemann Zeta-Function. The Theory of the Riemann Zeta-Function with Applications, Wiley, New York, 1985.

[12] M. Jutila, On Linnik's constant, Math. Scand. 41 (1975), 45-62.

[13] H. L. Montgomery, Topics in Multiplicative Number Theory, Lecture Notes in Math. 227, Springer, Berlin, 1971.

[14] H. L. Montgomery, A. Selberg, Linnik's theorem, unpublished.

[15] Y. Motohashi, On Linnik's zero-density theorem (Japanese), in: Characteristics of Arithmetic Functions (Proc. Sympos., Res. Inst. Math. Sci., Kyoto Univ., Kyoto, 1975), Sûrikaisekikenkyûsho Kókyûroku 274 (1976), 152-177.

[16] K. Prachar, Primzahlverteilung (German), Springer, Berlin, 1957.

[17] W. Wang, On the distribution of zeros of Dirichlet's L-functions (Chinese), J. Shangdong Univ., Nat. Sci. Ed. 21 (1986), No. 3, 1-13. 